Pacific Journal of Mathematics

INEQUALITIES FOR EIGENVALUES OF THE BIHARMONIC

Norman Mile AND R. Z. Ye H 


\title{
INEQUALITIES FOR EIGENVALUES OF THE BIHARMONIC OPERATOR
}

\author{
G. N. HILE AND R. Z. YeH
}

Let $D$ be a bounded domain in $R^{m}$ with smooth boundary. The first $n+1$ eigenvalues for the problem

$$
\Delta^{2} u-\mu u=0 \quad \text { in } D, \quad u=\frac{\partial u}{\partial n}=0 \quad \text { on } \partial D
$$

satisfy the inequality

$$
\sum_{i=1}^{n} \frac{\sqrt{\mu_{t}}}{\mu_{n+1}-\mu_{i}} \geq \frac{m^{2} n^{3 / 2}}{8(m+2)}\left(\sum_{i=1}^{n} \mu_{l}\right)^{-1 / 2}
$$

For the first two eigenvalues we have the stronger bound

$$
\begin{array}{ll}
\mu_{2} \leq 7.103 \mu_{1} & \left(\text { in } R^{2}\right), \\
\mu_{2} \leq 4.792 \mu_{1} & \left(\text { in } R^{3}\right) .
\end{array}
$$

The first two eigenvalues for the problem

$$
\Delta^{2} u+\nu \Delta u=0 \quad \text { in } D, \quad u=\frac{\partial u}{\partial n}=0 \quad \text { on } \partial D
$$

satisfy the inequality

$$
\begin{array}{ll}
\nu_{2} \leq 2.5 \quad \nu_{1} & \left(\text { in } R^{2}\right) \\
\nu_{2} \leq 2.12 \nu_{1} & \left(\text { in } R^{3}\right) .
\end{array}
$$

Introduction. Let $D$ be a bounded domain in $R^{m}, m \geq 2$, with smooth boundary $\partial D$. For the case $m=2$, Payne, Polya and Weinberger [6] obtained upper estimates, independent of the domain, for eigenvalues of the three well-known eigenvalue problems:

$$
\begin{aligned}
& \Delta u+\lambda u=0 \quad \text { in } D, \quad u=0 \\
& \text { on } \partial D \text {, } \\
& \Delta^{2} u-\mu u=0 \quad \text { in } D, \quad u=\frac{\partial u}{\partial n}=0 \quad \text { on } \partial D \text {, } \\
& \Delta^{2} u+\nu \Delta u=0 \quad \text { in } D, \quad u=\frac{\partial u}{\partial n}=0 \quad \text { on } \partial D \text {. }
\end{aligned}
$$

Let

$$
\begin{aligned}
& 0<\lambda_{1}<\lambda_{2} \leq \lambda_{3} \leq \cdots \\
& 0<\mu_{1} \leq \mu_{2} \leq \mu_{3} \leq \cdots \\
& 0<\nu_{1} \leq \nu_{2} \leq \nu_{3} \leq \cdots
\end{aligned}
$$


denote the successive eigenvalues for (1), (2) and (3), respectively. Payne, Polya and Weinberger showed that for domains in the plane,

$$
\begin{aligned}
\lambda_{n+1} \leq \lambda_{n}+\frac{2}{n} \sum_{i=1}^{n} \lambda_{i} & \leq 3 \lambda_{n}, \quad n=1,2, \ldots, \\
\mu_{n+1} \leq \mu_{n}+\frac{8}{n} \sum_{i=1}^{n} \mu_{l} \leq 9 \mu_{n}, & n=1,2, \ldots, \\
\nu_{2} \leq 3 \nu_{1} . &
\end{aligned}
$$

Inequality (4) was improved and extended to $m \geq 2$ by Protter and Hile [4]. They showed that the eigenvalues for (1) satisfy the inequality

$$
\sum_{i=1}^{n} \frac{\lambda_{i}}{\lambda_{n+1}-\lambda_{l}} \geq \frac{m n}{4}
$$

which amounts to an implicit bound for $\lambda_{n+1}$ in terms of the preceding eigenvalues. One can derive (4) from (7) by replacing each $\lambda_{l}$ in the denominators of the left-hand side of (7) by the larger quantity $\lambda_{n}$ and then solving the resulting inequality for $\lambda_{n+1}$.

In this paper we derive an analogue of (7) for the biharmonic eigenvalue problem (2). We obtain the implicit bound

$$
\sum_{l=1}^{n} \frac{\sqrt{\mu_{l}}}{\mu_{n+1}-\mu_{i}} \geq \frac{m^{2} n^{3 / 2}}{8(m+2)}\left(\sum_{l=1}^{n} \mu_{l}\right)^{-1 / 2} .
$$

From this inequality one may derive the weaker, but explicit, bound

$$
\mu_{n+1} \leq \mu_{n}+\frac{8(m+2)}{m^{2} n^{3 / 2}}\left(\sum_{i=1}^{n} \mu_{l}\right)^{1 / 2}\left(\sum_{i=1}^{n} \sqrt{\mu_{l}}\right),
$$

which in turn may be used to derive the still weaker, but perhaps aesthetically more appealing, bound

$$
\mu_{n+1} \leq \mu_{n}+\frac{8(m+2)}{m^{2} n} \sum_{i=1}^{n} \mu_{l}
$$

The last inequality (10) is the natural extension to dimensions $m \geq 2$ of the bound (5) for domains in the plane.

We also obtain improved estimates for some of the lower eigenvalues of (2). We show that for any $\sigma>0$,

$$
\mu_{n+1} \leq(1+\sigma) \mu_{n}+q(\sigma) \frac{M(m)}{n} \sum_{i=1}^{n} \mu_{l}
$$


where

$$
q(\sigma)=\left[\frac{(1+\sigma)^{3}}{\sigma}\right]^{1 / 2}, \quad M(m)=\frac{32}{3} \sqrt{\frac{2}{3}} m^{-1}(m+2)^{-1 / 2} .
$$

For given values of $m$ and $n$ one is obliged to select an optimal value of $\sigma$ so that the right side of (11) is minimized. For example, for $m=2, n=1$, one chooses $\sigma=.4$ to obtain the bound

$$
\mu_{2} \leq 7.103 \mu_{1} \quad\left(\text { in } R^{2}\right) \text {. }
$$

For $m=3, n=1$, the choice $\sigma=.36$ gives

$$
\mu_{2} \leq 4.792 \mu_{1} \quad\left(\text { in } R^{3}\right),
$$

and for $m=2, n=2$, we may choose $\sigma=.34$ to obtain

$$
\mu_{3} \leq 2.897 \mu_{1}+4.237 \mu_{2} \quad\left(\text { in } R^{2}\right) \text {. }
$$

The corresponding inequalities obtained from (10) are

$$
\begin{gathered}
\mu_{2} \leq 9 \mu_{1} \quad\left(\text { in } R^{2}\right), \quad \mu_{2} \leq 5.4 \overline{4} \mu_{1} \quad\left(\text { in } R^{3}\right) \\
\mu_{3} \leq 4 \mu_{1}+5 \mu_{2} \quad\left(\text { in } R^{2}\right) .
\end{gathered}
$$

Thus (11) is an improvement over (10) in these cases. We will show further that (11) is an improvement over (10) for $n=1$ and any value of $m$, and that for $m=2,3,4,(11)$ is stronger than (10) for $n \leq 8,3,2$, respectively.

In the last section of the paper we give an improvement and an extension to higher dimensions of inequality (6) for the eigenvalue problem (3). We show that

$$
\nu_{2} \leq \frac{m^{2}+8 m+20}{(m+2)^{2}} \nu_{1}
$$

Thus for $m=2,3$, for example, we have

$$
\begin{array}{ll}
\nu_{2} \leq 2.5 \nu_{1} & \left(\text { in } R^{2}\right), \\
\nu_{2} \leq 2.12 \nu_{1} & \left(\text { in } R^{3}\right) .
\end{array}
$$

We remark that there has been quite a bit of interest in obtaining the best estimate for the ratio $\lambda_{2} / \lambda_{1}$ for domains in the plane. Payne, Polya and Weinberger showed that

$$
\lambda_{2} \leq 3 \lambda_{1} \text {. }
$$

This bound has undergone a succession of improvements by Brands [1], DeVries [3], and Chiti [2], with the latest bound by Chiti being

$$
\lambda_{2} \leq 2.586 \lambda_{1} \text {. }
$$


For the circular disk we have $\lambda_{2} / \lambda_{1}=2.539 \cdots$. It has been conjectured [6], but not yet proved, that among all domains in the plane the circular disk maximizes the ratio $\lambda_{2} / \lambda_{1}$.

There has also been some interest in bounds for the quantities $\lambda_{3} / \lambda_{1}$, $\left(\lambda_{2}+\lambda_{3}\right) / \lambda_{1}$. See for example [1], [4], [5], [6]. The best bounds to date appear to be those of Marcellini [5], who showed that for domains in the plane,

$$
\lambda_{2}+\lambda_{3} \leq 5.596 \lambda_{1}, \quad \lambda_{3} \leq 3.917 \lambda_{1}
$$

1. Inequalities for $\mu_{n}$. Let $D$ be a bounded domain in $R^{m}, m \geq 2$, with boundary $\partial D$. Let the eigenvalues of

$$
\begin{gathered}
\Delta^{2} u=\mu u \quad \text { in } D, \\
u=\frac{\partial u}{\partial n}=0 \quad \text { on } \partial D
\end{gathered}
$$

be designated by

$$
0<\mu_{1} \leq \mu_{2} \leq \cdots \leq \mu_{n} \leq \cdots,
$$

with corresponding eigenfunctions $u_{1}, u_{2}, \ldots, u_{n}, \ldots$, normalized so that

$$
\int_{D} u_{i} u_{j}=\delta_{i j}, \quad i, j=1,2, \ldots
$$

The following theorem is an extension to higher dimensions of the inequalities (5). Later we will refine some of the techniques in the proof in order to obtain stronger inequalities.

THEOREM 1. For $m \geq 2$ and $n \geq 1$ we have

$$
\mu_{n+1} \leq \mu_{n}+\frac{8(m+2)}{m^{2} n} \sum_{i=1}^{n} \mu_{l} \leq\left(\frac{m+4}{m}\right)^{2} \mu_{n} \text {. }
$$

Proof. Following Payne, Polya and Weinberger [6] we consider the $n$ trial functions

$$
\varphi_{l}=x_{1} u_{i}-\sum_{j=1}^{n} a_{i j} u_{j}, \quad i=1,2, \ldots, n,
$$

where $x=\left(x_{1}, \ldots, x_{m}\right) \in R^{m}$, and the constants $a_{i j}$ are defined by

$$
a_{i j}=\int_{D} x_{1} u_{i} u_{j}, \quad i, j=1, \ldots, n
$$


Then each $\varphi_{i}$ is orthogonal to $u_{1}, \ldots, u_{n}$. Moreover, since $\varphi_{i}=\partial \varphi_{i} / \partial n=0$ on $\partial D$, we have the well-known inequality

$$
\mu_{n+1} \leq \frac{\int \varphi_{i} \Delta^{2} \varphi_{i}}{\int \varphi_{i}^{2}}, \quad i=1,2, \ldots, n
$$

Now,

$$
\begin{aligned}
\int \varphi_{i} \Delta^{2} \varphi_{i} & =\int \varphi_{i}\left[\Delta^{2}\left(x_{1} u_{i}\right)-\sum_{j=1}^{n} a_{i j} \mu_{j} u_{j}\right] \\
& =\int \varphi_{i}\left[x_{1} \Delta^{2} u_{i}+4 \Delta u_{i x_{1}}\right]=\mu_{i} \int \varphi_{i}^{2}+4 \int \varphi_{i} \Delta u_{i x_{1}}
\end{aligned}
$$

After substituting into (1.2) and summing over $i$ we have

$$
\mu_{n+1} \sum_{i=1}^{n} \int \varphi_{i}^{2} \leq \sum_{i=1}^{n} \mu_{i} \int \varphi_{i}^{2}+4 \sum_{i=1}^{n} \int \varphi_{i} \Delta u_{i x_{1}} .
$$

Now we make the assumption that

$$
\sum_{i=1}^{n} \int u_{i x_{k}}^{2}=\frac{1}{m} \sum_{i=1}^{n} \int\left|\nabla u_{l}\right|^{2}, \quad k=1, \ldots, m .
$$

This equality can be made to hold by rotating the coordinate system in $R^{m}$. Suppose, for example, that (1.5) does not hold, and that $x_{p}$ and $x_{q}$ denote two coordinate directions such that

$$
\sum_{i=1}^{n} \int u_{i x_{p}}^{2}<\frac{1}{m} \sum_{i=1}^{n} \int\left|\nabla u_{i}\right|^{2}<\sum_{i=1}^{n} \int u_{i x_{q}}^{2}
$$

Then we may make a rotation of the $x_{p}-x_{q}$ plane until (1.5) holds for, say, $k=p$, with the left-hand side of (1.5) remaining unaffected for values of $k$ different from $p$ and $q$. This operation can be repeated until (1.5) holds for all values of $k$.

We pause to make a few technical calculations. Let us define

$$
J_{1}=\sum_{i=1}^{n} \int \varphi_{l} \Delta u_{i x_{1}}, \quad J=\frac{m+2}{2 m} \sum_{i=1}^{n} \int\left|\nabla u_{i}\right|^{2} .
$$

LEMMA 1. The quantities $J_{1}$ and $J$ satisfy:

(i) $J_{1}=J$,

(ii) $J^{2} \leq n((m+2) / 2 m)^{2} \sum_{i=1}^{n} \mu_{i}$,

(iii) $n^{2}(m+2) / 8 \leq J \sum_{i=1}^{n} \int \varphi_{i}^{2}$. 
Proof of Lemma 1. (i) We have

$$
J_{1}=\sum_{i=1}^{n} \int x_{1} u_{l} \Delta u_{i x_{1}}-\sum_{i, j=1}^{n} a_{l j} \int u_{j} \Delta u_{i x_{1}} .
$$

The last term above vanishes, since $a_{i j}=a_{j l}$, and, by integration by parts,

$$
\int u_{J} \Delta u_{i x_{1}}=-\int u_{l} \Delta u_{j x_{1}}
$$

As for the first term, we show also by integration by parts that

$$
\begin{aligned}
\int x_{1} u_{i} \Delta u_{i x_{1}} & =\int \Delta\left(x_{1} u_{i}\right) u_{i x_{1}}=\int\left(x_{1} \Delta u_{i}+2 u_{l x_{1}}\right) u_{l x_{1}} \\
& =-\int\left(x_{1} \Delta u_{i}\right)_{x_{1}} u_{l}+2 \int u_{t x_{1}}^{2} \\
& =-\int x_{1} u_{l} \Delta u_{i x_{1}}+\int\left|\nabla u_{l}\right|^{2}+2 \int u_{i x_{1}}^{2} .
\end{aligned}
$$

Transposing, summing over $i$, and applying (1.5) we have

$$
J_{1}=\sum_{i=1}^{n}\left[\frac{1}{2} \int\left|\nabla u_{i}\right|^{2}+\int u_{l x_{1}}^{2}\right]=J \text {. }
$$

(ii) By the Schwarz inequality we have

$$
\begin{aligned}
J^{2} & =\left(\frac{m+2}{2 m}\right)^{2}\left(\sum_{l=1}^{n} \int-u_{i} \Delta u_{l}\right)^{2} \\
& \leq\left(\frac{m+2}{2 m}\right)^{2}\left(\sum_{l=1}^{n} \int u_{l}^{2}\right)\left(\sum_{i=1}^{n} \int\left(\Delta u_{i}\right)^{2}\right) .
\end{aligned}
$$

Moreover,

$$
\int u_{l}^{2}=1, \quad \int\left(\Delta u_{i}\right)^{2}=\int u_{i} \Delta^{2} u_{i}=\mu_{l} \int u_{i}^{2}=\mu_{i} .
$$

(iii) Let us compute

$$
\sum_{i=1}^{n} \int \varphi_{i} u_{i x_{1}}=\sum_{i=1}^{n} \int x_{1} u_{l} u_{i x_{1}}-\sum_{l, j=1}^{n} a_{l j} \int u_{j} u_{l x_{1}} .
$$

We have, by integration by parts

$$
\int x_{1} u_{l} u_{i x_{1}}=-\frac{1}{2}, \quad \int u_{j} u_{l x_{1}}=-\int u_{l} u_{j x_{1}} .
$$


Since $a_{i j}=a_{j i}$, the last term of (1.6) vanishes, and we obtain

$$
\sum_{l=1}^{n} \int \varphi_{l} u_{l x_{1}}=-\frac{n}{2}
$$

Hence, upon squaring and applying (1.5) we have

$$
\frac{n^{2}}{4} \leq\left(\sum_{l=1}^{n} \int \varphi_{l}^{2}\right)\left(\sum_{i=1}^{n} \int u_{i x_{1}}^{2}\right)=\left(\sum_{i=1}^{n} \int \varphi_{l}^{2}\right)\left(\frac{2}{m+2} J\right),
$$

which completes the proof of Lemma 1.

Returning now to (1.4), if we replace $\mu_{i}$ by $\mu_{n}$, then in view of Lemma 1 we have

$$
\left(\mu_{n+1}-\mu_{n}\right) \sum_{i=1}^{n} \int \varphi_{l}^{2} \leq 4 J .
$$

Moreover, combination of (ii) and (iii) of Lemma 1 yields

$$
J \leq \frac{2(m+2)}{n m^{2}}\left(\sum_{i=1}^{n} \mu_{l}\right)\left(\sum_{i=1}^{n} \int \varphi_{i}^{2}\right) .
$$

Substitution of (1.9) into (1.8) yields

$$
\mu_{n+1} \leq \mu_{n}+\frac{8(m+2)}{m^{2} n} \sum_{i=1}^{n} \mu_{l} \leq\left(\frac{m+4}{m}\right)^{2} \mu_{n},
$$

the last inequality being obtained by replacing $\mu_{i}$ by $\mu_{n}$ and simplifying. The proof of Theorem 1 is now complete.

Next we obtain a stronger result than Theorem 1 by a somewhat more lengthy argument.

THEOREM 2. For $m \geq 2$ and $n \geq 1$ we have the implicit bound

$$
\sum_{i=1}^{n} \frac{\sqrt{\mu_{i}}}{\mu_{n+1}-\mu_{i}} \geq \frac{m^{2} n^{3 / 2}}{8(m+2)}\left(\sum_{i=1}^{n} \mu_{i}\right)^{-1 / 2}
$$

and the explicit bound

$$
\mu_{n+1} \leq \mu_{n}+\frac{8(m+2)}{m^{2} n^{3 / 2}}\left(\sum_{i=1}^{n} \mu_{i}\right)^{1 / 2}\left(\sum_{i=1}^{n} \sqrt{\mu_{l}}\right) .
$$

Inequality (1.10) is stronger than (1.11), and both are stronger than (1.1). 
Proof. We return again to (1.4) but instead of replacing each $\mu_{i}$ by $\mu_{n}$ we introduce a new parameter $\alpha, \alpha>\mu_{n}$, and write

$$
\mu_{n+1} \sum_{i=1}^{n} \int \varphi_{i}^{2} \leq \alpha \sum_{i=1}^{n} \int \varphi_{i}^{2}+\sum_{i=1}^{n}\left(\mu_{i}-\alpha\right) \int \varphi_{i}^{2}+4 J .
$$

We also apply the Cauchy inequality to (1.7), obtaining for any $\delta>0$,

$$
\frac{n}{2} \leq \frac{\delta}{2} \sum_{i=1}^{n}\left(\alpha-\mu_{l}\right) \int \varphi_{t}^{2}+\frac{1}{2 \delta} \sum_{i=1}^{n}\left(\alpha-\mu_{i}\right)^{-1} \int u_{i x_{1}}^{2}
$$

Now we could use trial functions $\varphi_{i k}$, based on $x_{k}$ instead of $x_{1}$, for $k=1,2, \ldots, m$, and obtain inequalities analogous to (1.12) and (1.13) of the type

$$
\begin{aligned}
& \left(\mu_{n+1}-\alpha\right) \sum_{i=1}^{n} \int \varphi_{i k}^{2} \\
& \quad \leq \sum_{i=1}^{n}\left(\mu_{i}-\alpha\right) \int \varphi_{i k}^{2}+4 J, \quad k=1, \ldots, m, \\
& n \leq \delta \sum_{i=1}^{n}\left(\alpha-\mu_{i}\right) \int \varphi_{i k}^{2} \\
& \quad+\delta^{-1} \sum_{i=1}^{n}\left(\alpha-\mu_{i}\right)^{-1} \int u_{i x_{k}}^{2}, \quad k=1, \ldots, m .
\end{aligned}
$$

(Because of (1.5) and its consequence (i) of Lemma 1, the quantity " $J$ " is the same for each value of $k$.) We sum each of (1.14) and (1.15) over $k$, denoting

$$
S=\sum_{k=1}^{m} \sum_{i=1}^{n} \int \varphi_{i k}^{2}, \quad T=\sum_{k=1}^{m} \sum_{i=1}^{n}\left(\alpha-\mu_{i}\right) \int \varphi_{i k}^{2},
$$

to obtain the inequalities

$$
\begin{gathered}
\left(\mu_{n+1}-\alpha\right) S+T \leq 4 m J \\
m n \leq \delta T+\delta^{-1} \sum_{i=1}^{n}\left(\alpha-\mu_{i}\right)^{-1} \int\left|\nabla u_{i}\right|^{2} .
\end{gathered}
$$

We also have the estimate

$$
\int\left|\nabla u_{i}\right|^{2}=\int-u_{i}\left(\Delta u_{i}\right) \leq\left(\int u_{i}^{2}\right)^{1 / 2}\left(\int\left(\Delta u_{i}\right)^{2}\right)^{1 / 2}=\sqrt{\mu_{i}}
$$


which when applied to (1.17) yields

$$
m n \leq \delta T+\delta^{-1} \sum_{i=1}^{n} \frac{\sqrt{\mu_{i}}}{\alpha-\mu_{i}} .
$$

The right side of this inequality is minimized by choosing

$$
\delta=T^{-1 / 2}\left(\sum_{i=1}^{n} \frac{\sqrt{\mu_{i}}}{\alpha-\mu_{i}}\right)^{1 / 2} .
$$

Substitution of this value of $\delta$ into (1.18) and solving for $T$ yields

$$
T \geq \frac{m^{2} n^{2}}{4}\left(\sum_{i=1}^{n} \frac{\sqrt{\mu_{i}}}{\alpha-\mu_{i}}\right)^{-1}
$$

From (ii) of Lemma 1 we also have

$$
J \leq \sqrt{n} \frac{m+2}{2 m}\left(\sum_{i=1}^{n} \mu_{i}\right)^{1 / 2}
$$

Thus upon substituting (1.19) and (1.20) into (1.16) we arrive at the inequality

$$
\text { (1.21) }\left(\mu_{n+1}-\alpha\right) S \leq 2 \sqrt{n}(m+2)\left(\sum_{i=1}^{n} \mu_{i}\right)^{1 / 2}-\frac{m^{2} n^{2}}{4}\left(\sum_{i=1}^{n} \frac{\sqrt{\mu_{i}}}{\alpha-\mu_{i}}\right)^{-1} \text {. }
$$

Recall that $\alpha$ is restricted so that $\alpha>\mu_{n}$. We choose $\alpha$ so that the right side of (1.21) is zero. Thus

$$
\sum_{i=1}^{n} \frac{\sqrt{\mu_{i}}}{\alpha-\mu_{i}}=\frac{m^{2} n^{3 / 2}}{8(m+2)}\left(\sum_{i=1}^{n} \mu_{i}\right)^{-1 / 2} .
$$

Since the left side of (1.22) is a monotone decreasing function of $\alpha$ on $\left(\mu_{n}, \infty\right)$, decreasing from $+\infty$ to $0^{+}$, such a choice of $\alpha$ exists and in fact is unique. With this choice of $\alpha$ we obtain from (1.21) that $\mu_{n+1} \leq \alpha$. Thus it is clear that the replacement of $\alpha$ in (1.22) by $\mu_{n+1}$ increases the left-hand side. Hence we obtain (1.10).

Inequality (1.11) is derived from (1.10) by replacing each $\mu_{i}$ with $\mu_{n}$ in the denominators of the left-hand side, and then solving for $\mu_{n+1}$. Inequality (1.1) is in turn obtained from (1.11) by noting that

$$
\sum_{i=1}^{n} \sqrt{\mu_{i}} \leq \sqrt{n}\left(\sum_{i=1}^{n} \mu_{l}\right)^{1 / 2} .
$$

Thus the proof of Theorem 2 is complete. 
2. Stronger inequalities for lower eigenvalues. For the case $n=1$ the inequalities (1.1), (1.10), and (1.11) of $\$ 1$ all reduce to the same estimate for $\mu_{2}$, namely

$$
\mu_{2} \leq\left(\frac{m+4}{m}\right)^{2} \mu_{1}
$$

Thus in $R^{2}$ and $R^{3}$, for example, we have the estimates

$$
\mu_{2} \leq 9 \mu_{1} \quad\left(\text { in } R^{2}\right), \quad \mu_{2} \leq\left(\frac{7}{3}\right)^{2} \mu_{1} \doteq 5.445 \quad\left(\text { in } R_{3}\right)
$$

We will now obtain improvements of (2.1) for all values of $m$. In particular, we will show that

$$
\left.\mu_{2} \leq 7.103 \mu_{1} \quad\left(\text { in } R^{2}\right), \quad \mu_{2} \leq 4.792 \mu_{1} \quad \text { (in } R^{3}\right) \text {. }
$$

We will also obtain improved estimates for certain other lower eigenvalues in dimensions $m=2,3,4$. The method involves still further refinements of the arguments of $\S 1$. We retain the notation and terminology of the previous section.

The next theorem contains a partial improvement over Theorems 1 and 2 which will be stated explicitly in subsequent corollaries and theorems.

THEOREM 3. For $m \geq 2, n \geq 1$, and any constant $\sigma>0$ we have

$$
\begin{aligned}
\mu_{n+1} & \leq(1+\sigma) \mu_{n}+q(\sigma) \frac{M(m)}{n} \sum_{i=1}^{n} \mu_{i} \\
& \leq[1+\sigma+q(\sigma) M(m)] \mu_{n},
\end{aligned}
$$

where

$$
q(\sigma)=\left[\frac{(1+\sigma)^{3}}{\sigma}\right]^{1 / 2}, \quad M(m)=\frac{32}{3} \sqrt{\frac{2}{3}} m^{-1}(m+2)^{-1 / 2} .
$$

Proof. We begin again with inequality (1.4), apply (i) of Lemma 1, and introduce a real parameter $\beta$ to obtain

$$
\mu_{n+1} \sum_{i=1}^{n} \int \varphi_{i}^{2} \leq \sum_{i=1}^{n} \mu_{i} \int \varphi_{i}^{2}+4(1+\beta) J_{1}-4 \beta J
$$


We also introduce parameters $\sigma>0, \tau_{i}>0, i=1, \ldots, n$, and apply Cauchy's inequality to $J_{1}$ to obtain

$$
\begin{aligned}
4(1+\beta) J_{1} & =4(1+\beta) \sum_{i=1}^{n} \int-\nabla \varphi_{i} \cdot \nabla u_{i x_{1}} \\
& \leq \sum_{i=1}^{n} 2 \tau_{i} \int\left|\nabla \varphi_{i}\right|^{2}+2(1+\beta)^{2} \sum_{i=1}^{n} \tau_{i}^{-1} \int\left|\nabla u_{i x_{1}}\right|^{2} \\
& =\sum_{i=1}^{n} 2 \tau_{i} \int-\varphi_{i} \Delta \varphi_{i}+2(1+\beta)^{2} \sum_{i=1}^{n} \tau_{i}^{-1} \int u_{i x_{1} x_{1}} \Delta u_{l}
\end{aligned}
$$

But by Cauchy's inequality and (1.3) we have

$$
\begin{aligned}
\sum_{i=1}^{n} 2 \tau_{i} \int-\varphi_{l} \Delta \varphi_{i} & \leq \sum_{i=1}^{n} \sigma^{-1} \tau_{i}^{2} \int \varphi_{l}^{2}+\sum_{i=1}^{n} \sigma \int\left(\Delta \varphi_{i}\right)^{2} \\
& =\sum_{i=1}^{n}\left(\sigma \mu_{i}+\sigma^{-1} \tau_{l}^{2}\right) \int \varphi_{l}^{2}+4 \sigma J
\end{aligned}
$$

We substitute this expression into (2.4), and the resulting inequality into (2.3), and have as a result:

$$
\begin{aligned}
\mu_{n+1} \sum_{i=1}^{n} \int \varphi_{i}^{2} \leq & \sum_{i=1}^{n}\left(\mu_{i}+\sigma \mu_{i}+\sigma^{-1} \tau_{i}^{2}\right) \int \varphi_{i}^{2} \\
& +2(1+\beta)^{2} \sum_{i=1}^{n} \tau_{i}^{-1} \int u_{l x_{1} x_{1}} \Delta u_{i}+4(\sigma-\beta) J
\end{aligned}
$$

In order to simplify (2.5) we choose each $\tau_{l}$ so that

$$
(1+\sigma) \mu_{i}+\sigma^{-1} \tau_{i}^{2}=\tau, \quad i=1, \ldots, n,
$$

where $\tau$ is a new parameter. The conditions $\tau_{i}, \sigma>0$ require that $\tau>\mu_{n}$. In fact, we have

$$
\begin{gathered}
\tau>(1+\sigma) \mu_{n}>\mu_{n}, \\
\tau_{i}=\left[\tau-(1+\sigma) \mu_{i}\right]^{1 / 2} \sigma^{1 / 2}, \quad i=1, \ldots, n, \\
\tau_{1} \geq \tau_{2} \geq \cdots \geq \tau_{n}>0 .
\end{gathered}
$$

If we use trial functions $\varphi_{i k}$ based on $x_{k}$ instead of $x_{1}$, inequality (2.5) has the counterpart

$$
\begin{aligned}
\mu_{n+1} \sum_{i=1}^{n} \int \varphi_{l k}^{2} \leq & \tau \sum_{i=1}^{n} \int \varphi_{i k}^{2}+2(1+\beta)^{2} \sum_{i=1}^{n} \tau_{i}^{-1} \int u_{l x_{k} x_{k}} \Delta u_{i} \\
& +4(\sigma-\beta) J, \quad k=1, \ldots, m .
\end{aligned}
$$


We sum these inequalities over $k$, using

$$
\sum_{k=1}^{m} \int u_{i x_{k} x_{k}} \Delta u_{i}=\int\left(\Delta u_{\imath}\right)^{2}=\mu_{i}
$$

and obtain

$$
\left(\mu_{n+1}-\tau\right) S \leq 2(1+\beta)^{2} \sum_{i=1}^{n} \frac{\mu_{i}}{\tau_{i}}+4 m(\sigma-\beta) J .
$$

The counterpart of (iii) of Lemma 1 for $x_{k}$ is

$$
\frac{n^{2}(m+2)}{8} \leq J \sum_{i=1}^{n} \varphi_{i k}^{2}, \quad k=1, \ldots, m .
$$

Summing over $k$ leads to

$$
\frac{n^{2} m(m+2)}{8} \leq J S
$$

By restricting $\sigma-\beta<0$ we can use (2.8) to eliminate $J$ in (2.7), and then multiply by $S$ to obtain

$$
\left(\mu_{n+1}-\tau\right) S^{2}-2(1+\beta)^{2} \sum_{i=1}^{n} \frac{\mu_{l}}{\tau_{i}} S+\frac{m^{2}}{2}(m+2) n^{2}(\beta-\sigma) \leq 0 .
$$

Therefore, we have a quadratic inequality in $S$ of the form

$$
a S^{2}-2 b S+c \leq 0 \text {. }
$$

We can assert that

$$
a \leq b^{2} c^{-1},
$$

for unless $a \leq 0$, in which case we are done, the quadratic equation $a x^{2}-2 b x+c=0$ must have a real root. Thus

$$
\mu_{n+1}-\tau \leq \frac{2(1+\beta)^{4}}{m^{2}(m+2) n^{2}(\beta-\sigma)}\left(\sum_{i=1}^{n} \frac{\mu_{i}}{\tau_{i}}\right)^{2} .
$$

Since $f(\beta)=(1+\beta)^{4} /(\beta-\sigma)$ is minimized by taking $\beta=$ $(4 \sigma+1) / 3$ we substitute this value in the preceding inequality to obtain

$$
\mu_{n+1} \leq \tau+\frac{2\left(4^{4}\right)(1+\sigma)^{3}}{3^{3} m^{2}(m+2) n^{2}}\left(\sum_{i=1}^{n} \frac{\mu_{i}}{\tau_{i}}\right)^{2} .
$$

Since $\tau_{1} \geq \tau_{2} \geq \cdots \geq \tau_{n}$, replacing each $\tau_{i}$ by $\tau_{n}$ in (2.9) and further eliminating $\tau_{n}$ in favor of $\tau$ gives

$$
\mu_{n+1} \leq \tau+\frac{2\left(4^{4}\right)(1+\sigma)^{3}}{3^{3} m^{2}(m+2) n^{2} \sigma}\left(\sum_{i=1}^{n} \mu_{i}\right)^{2}\left[\tau-(1+\sigma) \mu_{n}\right]^{-1}
$$


The right side of inequality (2.10) has the form

$$
\tau+A(\tau-B)^{-1}
$$

which is minimized by letting $\tau=\sqrt{A}+B>(1+\sigma) \mu_{n}$. Substitution of this value of $\tau$ into (2.10) gives the desired result (2.2). The weakened version of (2.2) is obtained by replacing each $\mu_{i}$ with $\mu_{n}$, thereby completing the proof of Theorem 3.

If in inequality (2.2) of Theorem 3 we take $\sigma=.4$ for $m=2, n=1$, and $\sigma=.34$ for $m=2, n=2$, we obtain the following corollary.

COROllaRY 1. For domains in $R^{2}$ we have

$$
\begin{aligned}
& \mu_{2} \leq 7.103 \mu_{1}, \\
& \mu_{3} \leq 2.897 \mu_{1}+4.237 \mu_{2} .
\end{aligned}
$$

The corresponding inequalities obtained from (1.1) of Theorem 1 are

$$
\mu_{2} \leq 9 \mu_{1}, \quad \mu_{3} \leq 4 \mu_{1}+5 \mu_{2} \text {. }
$$

From (1.11) of Theorem 2 we also obtain

$$
\mu_{3} \leq \mu_{2}+2 \sqrt{2}\left(\mu_{1}+\mu_{2}\right)^{1 / 2}\left(\sqrt{\mu_{1}}+\sqrt{\mu_{2}}\right) .
$$

Inequality (1.10) of Theorem 2 also yields a different bound for $\mu_{3}$ which is quite a bit more complicated.

General comparisons between Theorem 3 and Theorems 1 and 2 are difficult to make for general $m$ and $n$. We will compare only Theorems 3 and 1, and only for a few simple cases. We first show that for $\mu_{2}$ the best bound is given by Theorem 3 in all dimensions. We compare the two inequalities

$$
\begin{gathered}
\mu_{n+1} \leq\left(\frac{m+4}{m}\right)^{2} \mu_{n} \quad(\text { Theorem 1), } \\
\mu_{n+1} \leq[1+\sigma+q(\sigma) M(m)] \mu_{n} \quad \text { (Theorem 3). }
\end{gathered}
$$

Inequality (2.12) holds for all $\sigma>0$. Thus the best bound is obtained by choosing $\sigma$ so that the right-hand side is minimized. In general a closed form expression for the optimal $\sigma$ is difficult to attain, since one is required to solve a cubic equation. We will show nevertheless that this optimal $\sigma$ always yields a better bound in (2.12) than the bound (2.11).

THEOREM 4. For all $m \geq 2$ inequality (2.12) is strictly stronger than (2.11) provided that the optimal value of $\sigma$ is chosen. 
Proof. Let us denote

$$
H_{m}(\sigma)=1+\sigma+q(\sigma) M(m),
$$

where $q(\sigma)$ and $M(m)$ are as described in Theorem 3. It must be shown that for some $\sigma>0$ we have

$$
H_{m}(\sigma)<\left(\frac{m+4}{m}\right)^{2}
$$

or, equivalently, for some $\sigma>0$,

$$
B_{m}(\sigma)>1
$$

where we define

$$
B_{m}(\sigma)=\sigma^{-1}\left[8(m+2) m^{-2}-M(m) q(\sigma)\right] .
$$

One can show after some lengthy computations that the maximum of $B_{m}(\sigma)$ occurs at

$$
\sigma=\bar{\sigma}_{m}:=\left[\frac{3}{8}(m+2)^{3} m^{-2}-1\right]^{-1},
$$

where obviously $\bar{\sigma}_{m}>0$, and the corresponding maximum of $B_{m}(\sigma)$ is

$$
B_{m}\left(\bar{\sigma}_{m}\right)=m^{-4}\left[m^{4}+8 m^{2}+32 m+16\right]>1 .
$$

We denote by $\sigma_{m}$ the optimal value of $\sigma$ which minimizes $H_{m}(\sigma)$, and hence the right side of inequality (2.12). By programming a calculator we have estimated a few values of $H_{m}\left(\sigma_{m}\right)$, which we denote by $H_{m}$. We find

$$
\begin{gathered}
H_{2} \doteq 7.103, \quad H_{3} \doteq 4.792, \quad H_{4} \doteq 3.704 \\
H_{5} \doteq 3.081, \quad H_{6} \doteq 2.684, \quad H_{7} \doteq 2.409 \\
H_{8} \doteq 2.210, \quad H_{9} \doteq 2.058, \quad H_{10} \doteq 1.939 \\
H_{100} \doteq 1.081578, \quad H_{1000} \doteq 1.008015963
\end{gathered}
$$

These numbers $H_{m}$ give upper bounds for the ratios $\mu_{n+1} / \mu_{n}$ for domains in $R^{m}$.

Next we compare in some special cases the following two inequalities, also appearing respectively in Theorems 1 and 3:

$$
\begin{gathered}
\mu_{n+1} \leq \mu_{n}+\frac{8(m+2)}{m^{2} n} \sum_{i=1}^{n} \mu_{i} \\
\mu_{n+1} \leq(1+\sigma) \mu_{n}+q(\sigma) \frac{M(m)}{n} \sum_{i=1}^{n} \mu_{i}
\end{gathered}
$$


THEOREM 5. In the cases $m=2,3,4$ and $n \leq 8,3,2$, respectively, inequality (2.15) is strictly stronger than (2.14) provided that the optimal value of $\sigma$ is chosen.

Proof. The right-hand sides of (2.14) and (2.15) may be rewritten as

$$
\begin{gathered}
P_{m, n}=\frac{8(m+2)}{m^{2} n} \sum_{i=1}^{n-1} \mu_{i}+\left[\frac{8(m+2)}{m^{2} n}\right] \mu_{n}, \\
H_{m, n}(\sigma)=\frac{1}{n} q(\sigma) M(m) \sum_{l=1}^{n-1} \mu_{i}+\left[1+\sigma+\frac{1}{n} q(\sigma) M(m)\right] \mu_{n} .
\end{gathered}
$$

Clearly, for any $\sigma>0$ such that

$$
\sigma+\frac{1}{n} q(\sigma) M(m) \leq \frac{8(m+2)}{m^{2} n},
$$

we will have

$$
H_{m, n}(\sigma)<P_{m, n} \text {. }
$$

But (2.16) is equivalent to

$$
B_{m}(\sigma)=\sigma^{-1}\left[8(m+2) m^{-2}-M(m) q(\sigma)\right] \geq n .
$$

Now, from (2.13) we have

$$
B_{2}\left(\bar{\sigma}_{2}\right)=8, \quad B_{3}\left(\bar{\sigma}_{3}\right) \doteq 3.3, \quad B_{4}\left(\bar{\sigma}_{4}\right) \doteq 2.1 .
$$

Therefore, for example, for $m=4$, if we restrict $n \leq 2$, then (2.71) is valid for $\sigma=\bar{\sigma}_{4}$. Similar statements hold for $m=3, n \leq 3$, and for $m=2$, $n \leq 8$.

3. Inequality for $\nu_{2}$. In a similar setting as before, let $\nu_{1}, \nu_{2}$, with $\nu_{1} \leq \nu_{2}$, be the first two eigenvalues of

$$
\begin{gathered}
\Delta^{2} u+\nu \Delta u=0 \quad \text { in } D \\
u=\frac{\partial u}{\partial n}=0 \quad \text { on } \partial D
\end{gathered}
$$

where $D$ is a bounded domain in $R^{m}$ with sufficiently smooth boundary. The following theorem is a technical improvement and an extension to higher dimensions of a result in [6].

THEOREM 6. For $m \geq 2$ we have

$$
\nu_{2} \leq \frac{m^{2}+8 m+20}{(m+2)^{2}} \nu_{1} \text {. }
$$


In particular, we have

$$
\nu_{2} \leq 2.5 \nu_{1} \quad \text { if } m=2, \quad \nu_{2} \leq 2.12 \nu_{1} \quad \text { if } m=3 .
$$

Proof. Let $u=u_{1}$ be the eigenfunction corresponding to $\nu=\nu_{1}$, normalized so that

$$
\int_{D}|\nabla u|^{2}=1
$$

As in $\S 1$, we may rotate the coordinate system so that

$$
\int u_{x_{1}}^{2}=\int u_{x_{2}}^{2}=\cdots=\int u_{x_{m}}^{2}=\frac{1}{m} \int|\nabla u|^{2} .
$$

We may further perform a translation in order that

$$
\int x_{k}|\nabla u|^{2}=0, \quad k=1, \ldots, m
$$

We start with the well-known inequality

$$
\nu_{2} \leq \frac{\int \varphi \Delta^{2} \varphi}{\int|\nabla \varphi|^{2}}
$$

which is satisfied by any sufficiently smooth function $\varphi$ such that

$$
\begin{gathered}
\varphi=\frac{\partial \varphi}{\partial n}=0 \quad \text { on } \partial D \\
\int_{D} \nabla \varphi \cdot \nabla u=0 .
\end{gathered}
$$

Following the method of Payne, Polya and Weinberger [6], we choose as our trial function

$$
\varphi=x_{1} u,
$$

which clearly satisfies the above boundary condition, and also the orthogonality condition, since

$$
\int \nabla \varphi \cdot \nabla u=\int x_{1}|\nabla u|^{2}+\int u u_{x_{1}}=0+0
$$

in view of (3.3) and integration by parts. Hence $\varphi=x_{1} u$ satisfies (3.4).

We next calculate the denominator of (3.4):

$$
\int|\nabla \varphi|^{2}=\int x_{1}^{2}|\nabla u|^{2}+2 \int x_{1} u u_{x_{1}}+\int u^{2}=\int x_{1}^{2}|\nabla u|^{2} .
$$


As for the numerator of (3.4), we have

$$
\int \varphi \Delta^{2} \varphi=\int \varphi\left(x_{1} \Delta^{2} u+4 \Delta u_{x_{1}}\right)=-v \int \varphi x_{1}(\Delta u)+4 \int \varphi \Delta u_{x_{1}} .
$$

Also, by integration by parts and (3.5),

$$
\begin{aligned}
\int \varphi x_{1}(\Delta u) & =-\int \nabla\left(x_{1} \varphi\right) \cdot \nabla u \\
& =-\int x_{1}^{2}|\nabla u|^{2}-2 \int x_{1} u u_{x_{1}}=-\int|\nabla \varphi|^{2}+\int u^{2} .
\end{aligned}
$$

Thus,

$$
\int \varphi \Delta^{2} \varphi=\nu \int|\nabla \varphi|^{2}-\nu \int u^{2}+4 \int \varphi \Delta u_{x_{1}}
$$

We pause to make some technical calculations. We define

$$
I=\int \varphi \Delta u_{x_{1}}
$$

LEMMA 2. We have:

(i) $I=(m+2) / 2 m$,

(ii) $I^{2} \leq \int|\nabla \varphi|^{2} \int u_{x_{1} x_{1}}(\Delta u)$,

(iii) $\int u^{2} \geq \nu^{-1}$.

Proof of Lemma 2. To prove (ii), we write

$$
I=-\int \nabla \varphi \cdot \nabla u_{x_{1}}
$$

By the Schwarz inequality and integration by parts,

$$
I^{2} \leq \int|\nabla \varphi|^{2} \int\left|\nabla u_{x_{1}}\right|^{2}=\int|\nabla \varphi|^{2} \int u_{x_{1} x_{1}}(\Delta u) .
$$

To prove (i), we proceed from (3.7), using integration by parts and (3.2), (3.1), to obtain

$$
\begin{aligned}
I & =-\int\left(x_{1} \nabla u \cdot \nabla u_{x_{1}}+u u_{x_{1} x_{1}}\right) \\
& =\frac{1}{2} \int|\nabla u|^{2}+\int u_{x_{1}}^{2}=\frac{m+2}{2 m} \int|\nabla u|^{2}=\frac{m+2}{2 m} .
\end{aligned}
$$


For (iii), working on (3.1) we have

$$
\begin{aligned}
1 & =\int-u \Delta u=\left(\int-u \Delta u\right)^{2} \leq\left(\int u^{2}\right) \int(\Delta u)^{2} \\
& =\int u^{2}\left(\int u \Delta^{2} u\right)=\left(\int u^{2}\right) \nu \int|\nabla u|^{2}=\nu \int u^{2},
\end{aligned}
$$

which completes the proof of Lemma 2.

Returning to (3.4) and (3.6) we have, in view of Lemma 2,

$$
\begin{aligned}
\nu_{2} \int|\nabla \varphi|^{2} & \leq \int \varphi \Delta^{2} \varphi \leq \nu \int|\nabla \varphi|^{2}-\nu\left(\nu^{-1}\right)+4 I \\
& =\nu \int|\nabla \varphi|^{2}+\frac{m+4}{m} .
\end{aligned}
$$

Now from Lemma 2 we also obtain

$$
1=\frac{2 m}{m+2} I=\left(\frac{2 m}{m+2}\right)^{2} I^{2} \leq \frac{4 m^{2}}{(m+2)^{2}} \int|\nabla \varphi|^{2} \int u_{x_{1} x_{1}} \Delta u .
$$

Thus division of (3.8) by $\int|\nabla \varphi|^{2}$ leads to

$$
\nu_{2} \leq \nu+\frac{4 m(m+4)}{(m+2)^{2}} \int u_{x_{1} x_{1}} \Delta u
$$

Had we used trial functions $\varphi_{k}=x_{k} u$, we would have obtained likewise

$$
\nu_{2} \leq \nu+\frac{4 m(m+4)}{(m+2)^{2}} \int u_{x_{k} x_{k}} \Delta u, \quad k=1, \ldots, m .
$$

Summing these inequalities over $k$ we obtain

$$
\nu_{2} \leq \nu+\frac{4(m+4)}{(m+2)^{2}} \int(\Delta u)^{2}
$$

But, as we have seen,

$$
\int(\Delta u)^{2}=\nu
$$

Hence,

$$
\nu_{2} \leq\left[1+\frac{4(m+4)}{(m+2)^{2}}\right] \nu=\frac{m^{2}+8 m+20}{(m+2)^{2}} \nu_{1} .
$$

This completes the proof of Theorem 4 . 


\section{REFERENCES}

[1] J. J. A. Brands, Bounds for the ratios of the first three membrane eigenvalues, Arch. Rational Mech. Anal., 16 (1964), 265-268.

[2] G. Chiti, $A$ bound for the ratio of the first two eigenvalues of a membrane, to appear.

[3] H. L. DeVries, On the upperbound for the ratio of the first two membrane eigenvalues, Z. Nauturforsch. A., 22 (1967), 152-153.

[4] G. N. Hile and M. H. Protter, Inequalities for eigenvalues of the Laplacian, Indiana Univ. Math. J., 29 (1980), 523-538.

[5] P. Marcellini, Bounds for the third membrane eigenvalues, J. Differential Equations, 37 (1980), 438-443.

[6] L. E. Payne, G. Polya and H. F. Weinberger, On the ratio of consecutive eigenvalues, J. Math. and Physics, 35 (1956), 289-298.

Received April 13, 1982. The first author was partially supported by NSF Grant MCS 81-00801.

UNIVERSITY OF HAWAII

HONOLULU, HI 96822 



\section{PACIFIC JOURNAL OF MATHEMATICS \\ EDITORS}

DONALD BABBITT (Managing Editor)

University of California.

Los Angeles, CA 90024

Hugo Rossi

University of Utah

Salt Lake City, UT 84112

C. C. Moore and Arthur Ogus

University of California

Berkeley, CA 94720
J. DugundjI

Department of Mathematics

University of Southern California

Los Angeles, CA 90089-1113

R. FinN and H. Samelson

Stanford University

Stanford, CA 94305

\section{ASSOCIATE EDITORS}
R. ARENS
E. F. BECKENBACH
B. H. NeumanN
F. WOLF
K. YoshidA (1906-1982)

\section{SUPPORTING INSTITUTIONS}

UNIVERSITY OF ARIZONA

UNIVERSITY OF BRITISH COLUMBIA

CALIFORNIA INSTITUTE OF TECHNOLOGY

UNIVERSITY OF CALIFORNIA

MONTANA STATE UNIVERSITY

UNIVERSITY OF NEVADA. RENO

NEW MEXICO STATE UNIVERSITY

OREGON STATE UNIVERSITY
UNIVERSITY OF OREGON

UNIVERSITY OF SOUTHERN CALIFORNIA

STANFORD UNIVERSITY

UNIVERSITY OF HAWAII

UNIVERSITY OF TOKYO

UNIVERSITY OF UTAH

WASHINGTON STATE UNIVERSITY

UNIVERSITY OF WASHINGTON 


\section{Pacific Journal of Mathematics}

Vol. 112, No. $1 \quad$ January, 1984

Richard Blaine Barrar and Henry Loeb, Characterizing the divided difference weights for extended complete Tchebycheff systems $\ldots \ldots \ldots \ldots 1$

Harold Bennett and David John Lutzer, Generalized ordered spaces with

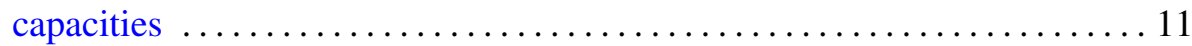

Geoffrey J. Butler and Lynn Harry Erbe, Comparison theorems for second-order operator-valued linear differential equations

Bohumil Cenkl and Richard D. Porter, de Rham theorem with cubical

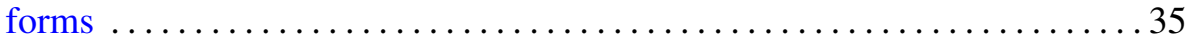

Zvonko Cerin, Characterizing global properties in inverse limits . ....... 49

Thomas Eugene Duchamp and Morris Kalka, Holomorphic foliations and deformations of the Hopf foliation .........................69 69

John Paul Hempel, Homology of coverings $\ldots \ldots \ldots \ldots \ldots \ldots \ldots \ldots \ldots$

Gerald Norman Hile and R. Z. Yeh, Inequalities for eigenvalues of the

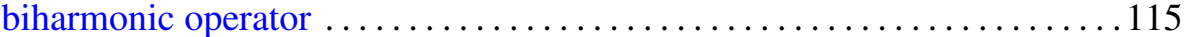

Kenneth Irwin Joy, A description of the topology on the dual space of a

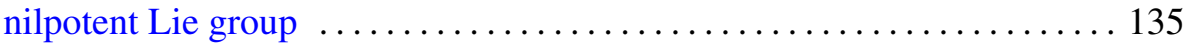

Alex Kumjian, On localizations and simple $C^{*}$-algebras $\ldots \ldots \ldots \ldots \ldots 141$

Bernardus de Pagter, The space of extended orthomorphisms in a Riesz

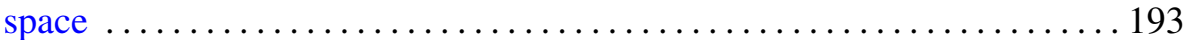

Stephen C. Persek, Iterated averaging for periodic systems with hidden

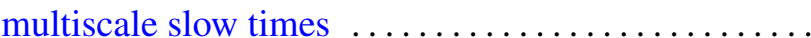

David Francis Rearick, Divisibility of arithmetic functions . . . . . . . . . 237

Masaaki Suzuki, The intrinsic metrics on the circular domains in $\mathbf{C}^{n}$ 